Supplement of Ann. Geophys., 38, 801-813, 2020

https://doi.org/10.5194/angeo-38-801-2020-supplement

(c) Author(s) 2020. This work is distributed under

the Creative Commons Attribution 4.0 License.

(c) (1)

Supplement of

\title{
BeiDa Imaging Electron Spectrometer observation of multi-period electron flux modulation caused by localized ultra-low-frequency waves
}

\section{Xingran Chen et al.}

Correspondence to: Qiugang Zong (qgzong@pku.edu.cn) and Hong Zou (hongzou@pku.edu.cn)

The copyright of individual parts of the supplement might differ from the CC BY 4.0 License. 

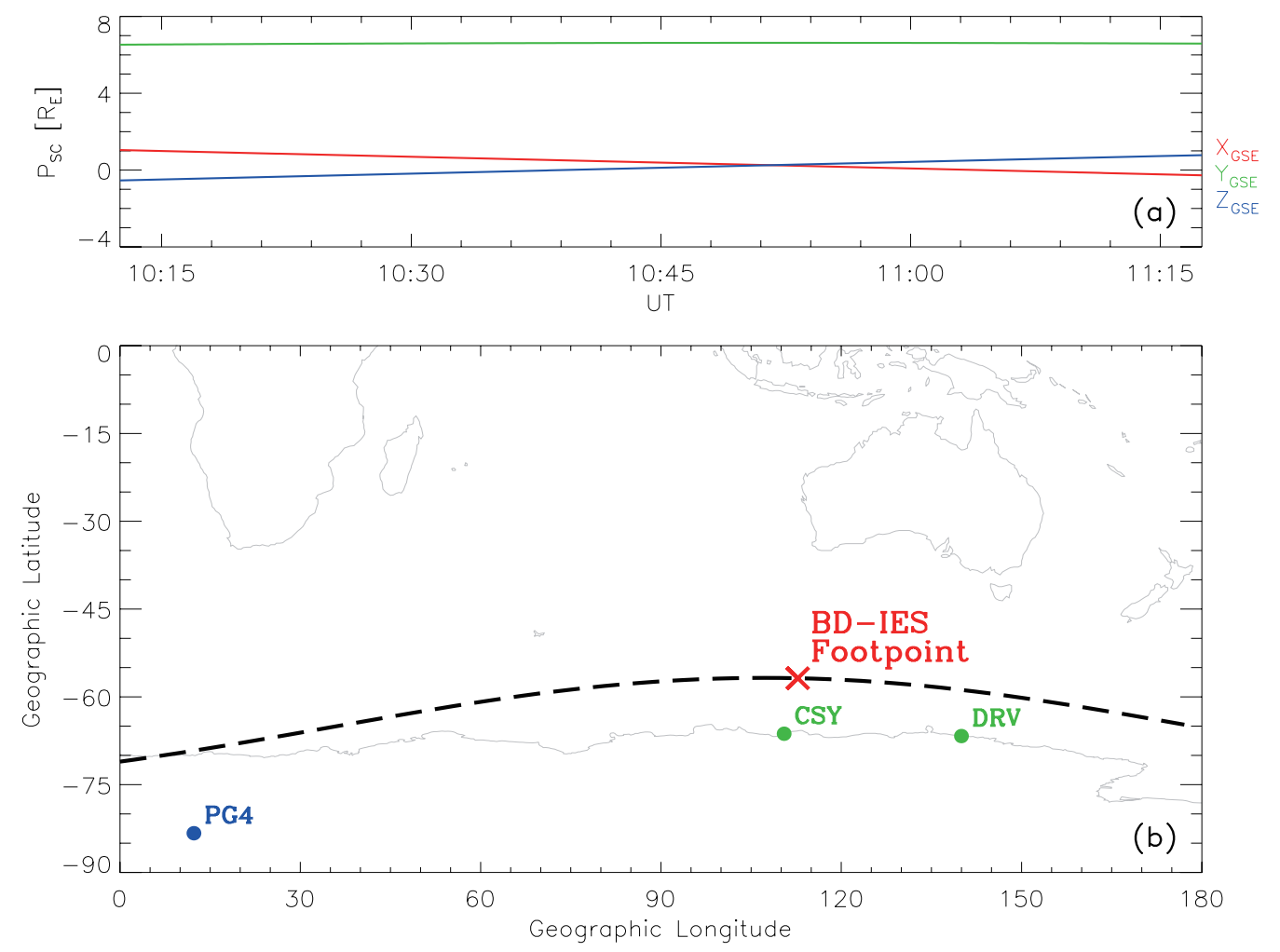

Figure S1: Locations of the spacecraft and the ground-based geomagnetic observatories. (a) Spacecraft position in GSE coordinates. (b) Footpoint of the spacecraft and locations of the three ground-based magnetometers. The black dashed line shows the isopleth of the geomagnetic latitude equivalent to the spacecraft footpoint. The two stations near the spacecraft footpoint, marked by the green dots, did not observe ULF oscillations while the station away from the footpoint, marked by the blue dot, observed ULF oscillations in the relevant periods (shown in Figure), which supports the idea that the ULF waves in our event was azimuthally confined and the spacecraft was located outside of wave active region. 


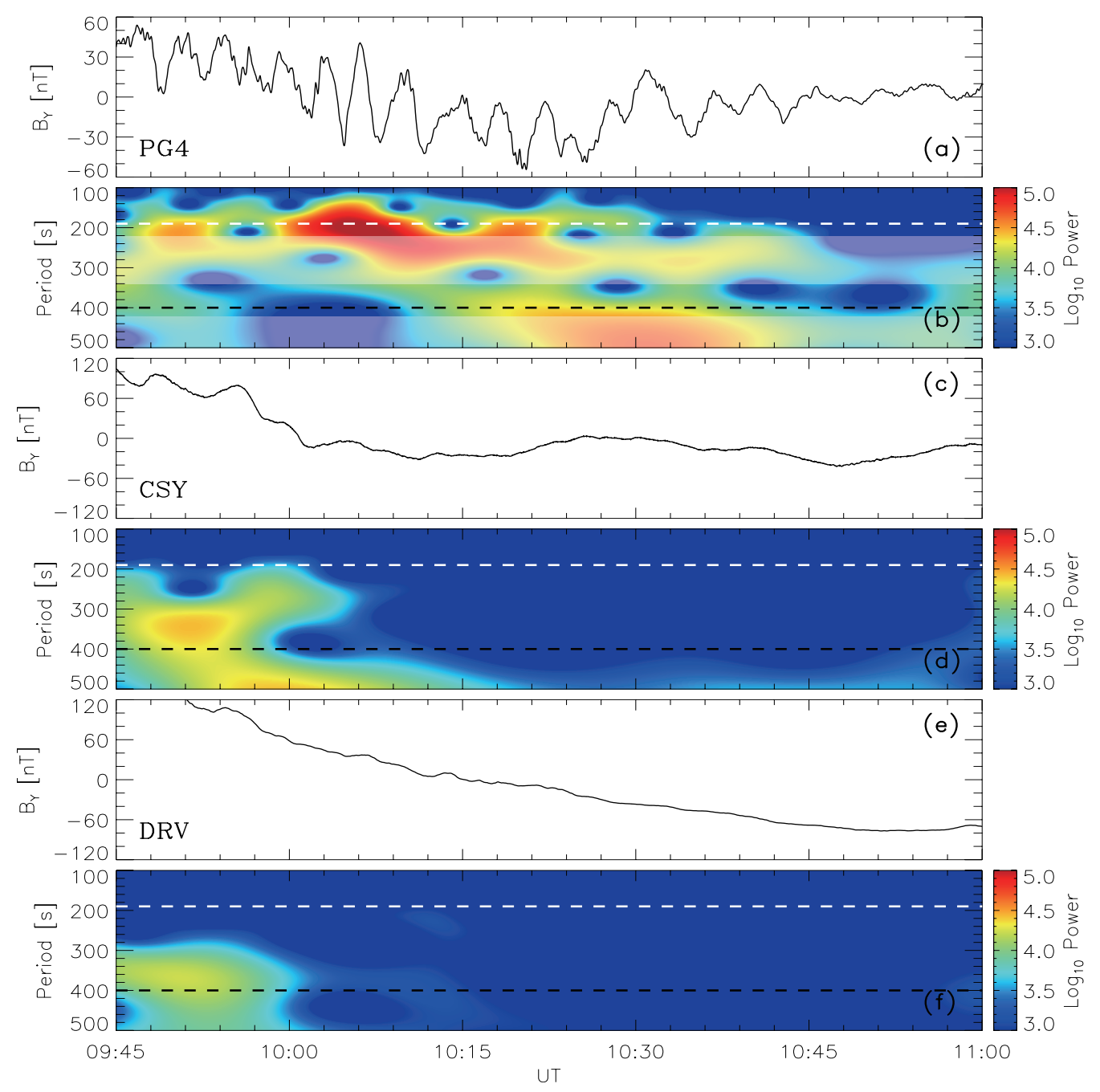

Figure S2: Ground-based magnetic field observations. $(a)(c)(e)$ The eastwest component of magnetic field observed at PG4, CSY, and DRV groundbased magnetometer stations. $(b)(d)(f)$ The corresponding wavelet power spectrogram. The horizontal dashed lines mark the periods of $190 \mathrm{~s}$ and 400 s. The two stations near the spacecraft footpoint, CSY and DRV, did not observe ULF perturbation, while oscillations in the Pc 3-5 band was apparently observed by PG4 away from the footpoint in the azimuthal direction. Since the position of PG4 mapped to a higher L shell, the eigen-frequency of the corresponding field lines are usually lower (e.g. Southwood, 1974; Kivelson and Southwood, 1985). Therefore, the magnetic field oscillations possibly associated with the wave-particle interactions might be observed at longer periods, which are shaded in panel $(b)$. 


\section{References}

Kivelson, M. G. and Southwood, D. J.: Resonant ULF waves: A new interpretation, Geophys. Res. Lett., 12, 49-52, doi:10.1029/GL012i001p00049, 1985.

Southwood, D. J.: Some features of field line resonances in the magnetosphere, Planet. Space Sci., 22(3), 483-491, 1974. 